\title{
Preferences of Chirality and Polarity in Triglycine sulfate Crystals
}

\section{Yukana Terasawa ${ }^{1}$, Toshio Kikuta ${ }^{2}$, Masaaki Ichiki $^{3}$, Sota Sato ${ }^{4}$, Kazuhiko Ishikawa $^{1}$, Toru Asahi ${ }^{5,6}$}

${ }^{1}$ School of Advance Science and Engineering, Waseda University, 2-2 Wakamatsu-cho Shinjuku-ku, Tokyo, 162-8480, Japan, ${ }^{2}$ Faculty of Engineering, University of Toyama, 3190 Gofuku, Toyama-shi, Toyama, 930-8555, Japan, ${ }^{3}$ Research Center for Ubiquitous MEMS and Micro Engineering, National Institute of Advanced Industrial Science and Technology (AIST), 1-2-1 namiki, Tsukuba-shi, Ibaraki, 305-8564, Japan, ${ }^{4}$ Department of Chemistry, The University of Tokyo, 7-3-1 Hongo, Bunkyo-ku, Tokyo, 113-0033, Japan, ${ }^{5}$ Faculty of Science and Engineering, Waseda University, 3-4-1 okubo, Shinjuku-ku, Tokyo, 169-8555, Japan, ${ }^{6}$ Research Organization for Nano \& Life Innovation, Waseda University, 513 Wasedatsurumaki-cho Shinjuku-ku,Tokyo, 162-0041, Japan

$$
\text { tinkerbell@akane.waseda.jp }
$$

Chirality is a property that real images are non-superimposable on their mirror images. The importance of chirality has commonly been known through drug incidents of thalidomide all over the world $1_{1,2}{ }^{2}$. Chirality exists not only molecules, crystals, membranes and other objects in nature. Crystal chirality is derived from not only molecular chirality but also helical arrangement of molecules in crystals. In the latter case, even if achiral molecules are put in a right-handed or a left-handed helical arrangement in crystals, the crystals occur chirality. It has already been known that the same amount of left-handed and right-handed crystals are obtained when chiral crystals composed of achiral molecules are grown $3^{3}$. Among crystals composed of achiral molecules, about $8 \%$ of them are chiral crystals, so it is very important to grow chiral crystals that have particular chirality. However, it is extremely difficult to grow only right-handed or left-handed crystals from achiral molecules. In this study, we succeeded in growing right-handed or left-handed crystals from achiral molecules.

We have focused on Triglycine sulfate (TGS) crystals composed of glycine and sulfuric acid (Figure 1(a)). We found that TGS with particular chirality has grown by doping with L-, or D-alanine (Figure 1(b)). L-alanine-doped TGS (LATGS) crystals showed lefthandedness, while D-alanine-doped TGS (DATGS) crystals showed right-handedness (Figure 2). This is an extremely interesting phenomenon. We discuss that this phenomenon is derived from polarity because TGS is ferroelectricity. The relationship between chirality and polarity helps the elucidation of the explicit mechanism of preferred chirality of TGS crystal by alanine.

(a)

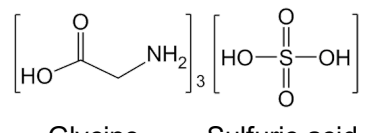

(b)

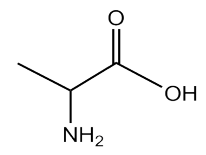

Figure 1. Molecular structure. (a) TGS. TGS is composed of achiral molecules, glycine and sulfuric acid. (b) Alanine. Molecular structure of Alanine is similar to that of glycine.

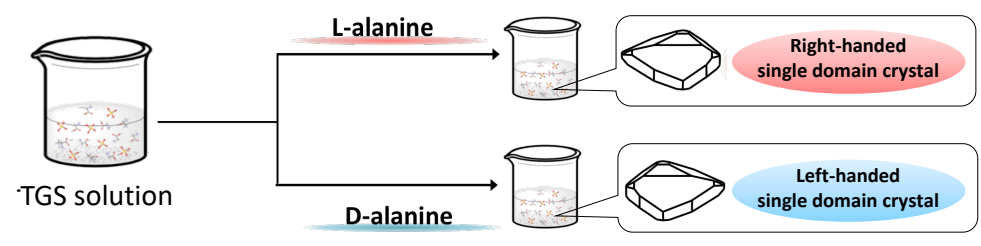

Figure 2. Chiral preference in TGS crystal by alanine.

[1] Blaschke, G., et. al., (1979). Arzneim..-forsch., 29, 1640-1642,

[2] Nishimura, K., et al., (1993). Chem. Pharm. Bull. 42, 1157-1159,

[3] Pasteur, L., (1848). Annales de Chemie et de Physique, 24, 442-459.

Keywords: chirality; polarity; ferroelectricity

Acta Cryst. (2021), A77, C1240 